\title{
Applications of the general Lyapunov ISS small-gain theorem for networks
}

\author{
Sergey N. Dashkovskiy
}

Björn S. Rüffer

\author{
Fabian R. Wirth
}

\begin{abstract}
In the framework of the ISS Lyapunov formulation a small gain theorem has recently been proved which allows the explicit construction of Lyapunov functions for interconnected systems. In this note we recall the definitions of ISS Lyapunov functions and the corresponding general small gain theorems. These are then exemplarily used to prove inputto-state stability of and to construct ISS Lyapunov functions for four areas of applications: Linear systems, a Cohen-Grossberg neuronal network, error dynamics in formation control, as well as nonlinear transistor-linear resistor circuits.
\end{abstract}

Index Terms - Input-to-state stability (ISS), Lyapunov functions, general small-gain theorem, constructive method, example applications

\section{INTRODUCTION}

For nonlinear systems it is often difficult to prove stability properties, especially if several such systems are in a feedback interconnection. Stability of interconnections of nonlinear systems can be studied in different frameworks such as passivity, dissipativity [18], input-to-state stability [19] and others. Knowledge of a Lyapunov function of a system makes stability analysis simple, but finding a Lyapunov function is an art of its own and generally also a complicated task, especially for higher dimensional systems.

One approach is to split complicated high dimensional systems into smaller, interconnected subsystems evolving in lower dimensional spaces. The idea is to find Lyapunov functions for the lower dimensional systems and then combine these together to obtain a Lyapunov function for the high dimensional system. Here it has to be taken into account that the subsystems themselves are interconnected in a nonlinear fashion and that there may exist external inputs or disturbances that have to be accounted for.

In this paper we use the input-to-state stability (ISS) framework introduced by Sontag [19]. Its equivalent Lyapunov characterization [20] and the corresponding small gain theorem [11] or its generalization to more than two interconnected systems [7], [9] can be implemented to establish exactly the idea detailed above: A bottom-to-top stability analysis of high dimensional nonlinear systems. See [8] for an application of these results in a logistic system.

We consider several example applications of general ISS small gain theorems provided by the authors in [3], [5], [7], [6], [9]. In particular we apply the nonlinear Lyapunov ISS

DSN is with the Center of Applied Mathematics, University of Bremen, Germany, dsnemath. uni-bremen. de

BSR is with the School of Electrical Engineering, and Computer Science, University of Newcastle, Australia, Bjoern.Rueffer@newcastle.edu.au

FRW is with the Institute of Mathematics, University of Würzburg, Germany, wirthemathematik.uni-wuerzburg.de small-gain theorem for networks to interconnected linear systems a Cohen-Grossberg neuronal network, error dynamics in formation control and the stability analysis of transistor networks.

The paper is organized as follows. In the next section we recall the necessary definitions and notation. In Section III we quote the small gain type theorems related to ISS networks. The main results of this paper are four example applications that are given in Section IV. Section V concludes the paper.

\section{NOTATION AND DEFINITIONS}

Consider several nonlinear systems with inputs given by

$$
\Sigma_{i}: \dot{x}_{i}=f_{i}\left(x_{1}, \ldots, x_{n}, u_{i}\right), \quad i=1, \ldots, n,
$$

where $x_{i} \in \mathbb{R}^{N_{i}}, u_{i} \in \mathbb{R}^{M_{i}}$. The functions $f_{i}$ : $\mathbb{R}^{\sum_{j} N_{j}+M_{i}} \rightarrow \mathbb{R}^{N_{i}}, i=1, \ldots, n$, are assumed to be such that there exists a unique solution for any given initial condition and any essentially bounded, measurable inputs $x_{j}, j \neq i, u_{i}$. We say that $x_{j}$ is an internal input for the system $\Sigma_{i}$ with $i \neq j$ and $u_{i}$ is an external input to $\Sigma_{i}$. Denoting $x=\left(x_{1}^{T}, \ldots, x_{n}^{T}\right)^{T} \in \mathbb{R}^{N}, N=\sum_{i=1}^{n} N_{i}, u=$

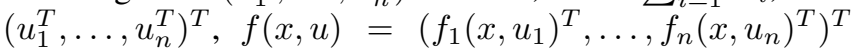
we can represent the interconnection in the form

$$
\dot{x}=f(x, u) \text {. }
$$

An appropriate stability notion for such systems is input-tostate stability as introduced in [19]. To recall the definition we will need the following notation. Let $\mathbb{R}_{+}:=[0, \infty)$; a function $\gamma: \mathbb{R}_{+} \rightarrow \mathbb{R}_{+}$is said to be of class $\mathcal{K}$ if it is continuous, increasing and $\gamma(0)=0$. It is of class $\mathcal{K}_{\infty}$ if, in addition, it is unbounded. A function $\beta: \mathbb{R}_{+} \times \mathbb{R}_{+} \rightarrow \mathbb{R}_{+}$ is said to be of class $\mathcal{K} \mathcal{L}$ if, for each fixed $t$, the function $\beta(\cdot, t)$ is of class $\mathcal{K}$ and, for each fixed $s$, the function $\beta(s, \cdot)$ is non-increasing and tends to zero at infinity. Let $|x|$ denote the Euclidean norm of $x \in \mathbb{R}^{n},\|\cdot\|$ be the induced operator norm and $\|\cdot\|_{\infty}$ be the standard norm in the space of essentially bounded functions $L_{\infty}$. By $\mathbb{R}_{+}^{n}$ we denote the positive orthant in $\mathbb{R}^{n}$. For two vectors $a$ and $b$ in $\mathbb{R}^{n}$ we denote $a \geq b \quad \Leftrightarrow \quad a_{i} \geq b_{i}, i=1, \ldots, n$. The relation $a>b$ is defined in the same way. The negation of the relation $a \geq b$ is denoted by $a \nsupseteq b$. This is equivalent to the statement that there exists at least one $i \in\{1, \ldots, n\}$ such that $a_{i}<b_{i}$. By id we will denote the identity map in an appropriate space.

Definition 2.1: System (2) is called input-to-state stable from $u$ to $x$, if there exists a $\gamma \in \mathcal{K}_{\infty}$, and a $\beta \in \mathcal{K} \mathcal{L}$, such that

$$
|x(t)| \leq \beta(|x(0)|, t)+\gamma\left(\|u\|_{\infty}\right) \quad \forall t \geq 0,
$$


for all initial states $x(0) \in \mathbb{R}^{N}$ and inputs $u \in L_{\infty}$. In this case $\gamma$ is called nonlinear gain.

It is known that ISS defined in this way is equivalent to the existence of an ISS-Lyapunov function defined by

Definition 2.2: A smooth function $V: \mathbb{R}^{N} \rightarrow \mathbb{R}_{+}$is called ISS-Lyapunov function of (2) if there exist $\psi_{1}, \psi_{2} \in$ $\mathcal{K}_{\infty}, \chi \in \mathcal{K}_{\infty}$, and a positive definite function $\alpha$ such that

$$
\begin{gathered}
\psi_{1}(|x|) \leq V(x) \leq \psi_{2}(|x|), \quad \forall x \in \mathbb{R}^{N}, \\
V(x) \geq \chi(|u|) \Longrightarrow \nabla V(x) \cdot f(x, u) \leq-\alpha(V(x)) .
\end{gathered}
$$

The function $\chi$ is then called Lyapunov gain.

In case of an interconnected system (1) there are possibly multiple nonlinear gains related to different inputs in each subsystem.

Typically one says that $\Sigma_{i}$ is ISS if there exist $\gamma_{i u}, \gamma_{i j} \in$ $\left(\mathcal{K}_{\infty} \cup\{0\}\right)$ and $\beta_{i} \in \mathcal{K} \mathcal{L}$ so that all solutions satisfy

$$
\begin{array}{r}
\left|x_{i}(t)\right|<\beta_{i}\left(\left|x_{i}\left(t_{0}\right)\right|, t-t_{0}\right)+\sum_{j \neq i} \gamma_{i j}\left(\left\|x_{j}\right\|_{\infty}\right) \\
+\gamma_{u i}\left(\left\|u_{i}\right\|_{\infty}\right), \quad t \geq 0 .
\end{array}
$$

Qualitatively equivalent, one could replace the sum $\sum_{j \neq i}$ with a maximization $\max _{j \neq i}$ or similar terms; depending on the application one formulation might be more natural and give tighter estimates than others. To accommodate all such possible formulations, we introduce the following general definition:

Definition 2.3: A continuous function $\mu: \mathbb{R}_{+}^{n} \rightarrow \mathbb{R}_{+}$is called a monotone aggregation function if it satisfies

(i) $\mu(0)=0$.

(ii) $\mu(s) \geq 0$ for all $s \in \mathbb{R}_{+}^{n}$ and $\mu(s)>0$ if $s>0$.

(iii) for any $s_{1}>s_{2} \in \mathbb{R}_{+}^{n}$ it holds that $\mu\left(s_{1}\right)>\mu\left(s_{2}\right)$.

The space of monotone aggregation functions is denoted by $\mathrm{MAF}_{n}$ and $\mathrm{MAF}_{n}^{m}$ denotes $m$-vectors of monotone aggregation functions, i.e., maps $\mu: \mathbb{R}_{+}^{m \times n} \rightarrow \mathbb{R}_{+}^{m}$.

As a convention we assume $\gamma_{i i} \equiv 0$ for $i=1, \ldots, n$. Using the MAF notation we say that $\Sigma_{i}$ is ISS if there exist $\mu_{i} \in$ $\mathrm{MAF}_{n+1}, \gamma_{i u}, \gamma_{i j} \in\left(\mathcal{K}_{\infty} \cup\{0\}\right)$ and $\beta_{i} \in \mathcal{K} \mathcal{L}$ so that all solutions satisfy

$$
\begin{array}{r}
\left|x_{i}(t)\right|<\mu_{i}\left(\beta_{i}\left(\left|x_{i}\left(t_{0}\right)\right|, t-t_{0}\right), \gamma_{i 1}\left(\left\|x_{1}\right\|_{\infty}\right),\right. \\
\left.\ldots, \gamma_{i n}\left(\left\|x_{n}\right\|_{\infty}\right), \gamma_{i u}\left(\|u\|_{\infty}\right)\right) .
\end{array}
$$

Similarly, we define the concept of ISS-Lyapunov functions. Assume that for each of the subsystems $\Sigma_{i}, i=1, \ldots, n$ we are given a proper, positive definite function $V_{i}: \mathbb{R}^{N_{i}} \rightarrow \mathbb{R}_{+}$. The function $V_{i}: \mathbb{R}^{N_{i}} \rightarrow \mathbb{R}_{+}$is an ISS-Lyapunov function for $\Sigma_{i}$ if there exist $\mu_{i} \in \mathrm{MAF}_{n+1}, \gamma_{i j} \in\left(\mathcal{K}_{\infty} \cup\{0\}\right), \gamma_{i u} \in$ $\mathcal{K}_{\infty}$ and a positive definite function $\alpha_{i}$ such that

$$
\begin{array}{r}
V_{i}\left(x_{i}\right) \geq \mu_{i}\left(\gamma_{i 1}\left(V_{1}\left(x_{1}\right)\right), \ldots, \gamma_{i n}\left(V_{n}\left(x_{n}\right)\right), \gamma_{i u}\left(\left|u_{i}\right|\right)\right. \\
\Longrightarrow \nabla V_{i}\left(x_{i}\right) f_{i}(x, u)<-\alpha\left(\left|x_{i}\right|\right),
\end{array}
$$

again with the convention $\gamma_{i i}=0$ for all $i$. The gains $\gamma_{i j}$ can be combined in a gain matrix $\Gamma:=\left(\gamma_{i j}\right)_{i, j=1, \ldots, n}$. If we include the gains $\gamma_{i u}$ in the last column of this matrix, then the obtained matrix is denoted by $\bar{\Gamma}$. The expressions in the above implication motivate the following definition of a nonlinear map

$$
\begin{aligned}
& \bar{\Gamma}_{\mu}: \mathbb{R}_{+}^{n+1} \rightarrow \mathbb{R}_{+}^{n} \\
& {\left[\begin{array}{c}
s_{1} \\
\vdots \\
s_{n} \\
r
\end{array}\right] \mapsto\left[\begin{array}{c}
\mu_{1}\left(\gamma_{11}\left(s_{1}\right), \ldots, \gamma_{1 n}\left(s_{2}\right), \gamma_{1 u}(r)\right) \\
\vdots \\
\mu_{n}\left(\gamma_{n 1}\left(s_{1}\right), \ldots, \gamma_{n n}\left(s_{n}\right), \gamma_{n u}(r)\right)
\end{array}\right]}
\end{aligned}
$$

The map $\Gamma_{\mu}$ is defined similarly by

$$
\begin{aligned}
& \Gamma_{\mu}: \mathbb{R}_{+}^{n+1} \rightarrow \mathbb{R}_{+}^{n} \\
& {\left[\begin{array}{c}
s_{1} \\
\vdots \\
s_{n} \\
r
\end{array}\right] \mapsto\left[\begin{array}{c}
\mu_{1}\left(\gamma_{11}\left(s_{1}\right), \ldots, \gamma_{1 n}\left(s_{2}\right), 0\right) \\
\vdots \\
\mu_{n}\left(\gamma_{n 1}\left(s_{1}\right), \ldots, \gamma_{n n}\left(s_{n}\right), 0\right)
\end{array}\right]}
\end{aligned}
$$

Even if each subsystem $\Sigma_{i}$ in (1) is ISS, their interconnection (2) can be unstable [5]. In the next section we recall the small gain type condition which guarantees ISS of the interconnection. We also recall the construction of an ISSLyapunov function for the interconnected system.

\section{Small Gain Theorems}

A stability condition of small gain type for arbitrary interconnection of a finite number of ISS systems has been obtained in [3], [5], [15]. For a Lyapunov version of this result see [4], [7], [9], [15]. The general small gain theorem and the corresponding construction of an ISS-Lyapunov function generalize known results for the interconnection of two ISS systems as in [12], [11]. We only quote the Lyapunov result that will be applied in the following section.

Theorem 3.1: Let $\Gamma \in\left(\mathcal{K}_{\infty} \cup\{0\}\right)^{n \times n}$ be a gain matrix and $\mu \in \operatorname{MAF}_{n}^{n}$. If there exists an $\alpha \in \mathcal{K}_{\infty}$ such that the small gain condition

$$
D \circ \Gamma_{\mu}(s) \nsupseteq s \quad \forall s \supsetneqq 0
$$

holds with $D:=\operatorname{diag}(\mathrm{id}+\alpha)$, then there exists a $\tilde{D}=$ $\operatorname{diag}(\mathrm{id}+\tilde{\alpha}), \tilde{\alpha} \in \mathcal{K}_{\infty}$ and a continuous path $\sigma \in \mathcal{K}_{\infty}^{n}$ with

(i) $\sigma$ is locally Lipschitz continuous on $(0, \infty)$ and in particular differentiable almost everywhere in $[0, \infty)$;

(ii) for every compact $K \subset(0, \infty)$ there are constants $0<c<C$ such that for all points of differentiability of $\sigma_{i}$ and $i=1, \ldots, n$ we have

$$
0<c \leq \sigma_{i}^{\prime}(r) \leq C
$$

(iii)

$$
\tilde{D}\left(\Gamma_{\mu}(\sigma(r))\right)<\sigma(r), \quad \forall r>0 .
$$

Motivated by the existence of a $\Gamma_{\mu}$-invariant decay set $\Omega \subset \mathbb{R}_{+}^{n}$ where the path parameterized by $\sigma$ evolves, in the sequel we will call a function $\sigma \in \mathcal{K}_{\infty}^{n}$ satisfying (11) an $\Omega$-path with respect to $\Gamma_{\mu}$. See [4], [7], [15], [16] for details concerning $\Omega$.

We note that condition (10) guarantees, that $\sigma$ is locally bi-Lipschitz on $(0, \infty)$. In the following result we see, that regularity properties of the inverse component functions are needed to obtain a Lyapunov function. 
Theorem 3.2: Consider the interconnected system $\Sigma$ given by (1), (2) where for each of the subsystems $\Sigma_{i}$ there exists an ISS-Lyapunov function $V_{i}$ and the corresponding gain matrix defines a map $\bar{\Gamma}_{\mu}$ as in (7). Assume there are functions $\sigma=\left(\sigma_{1}, \ldots, \sigma_{n}\right) \in \mathcal{K}_{\infty}^{n}, \varphi \in \mathcal{K}_{\infty}$ such that

$$
\bar{\Gamma}_{\mu}(\sigma(r), \varphi(r))<\sigma(r), \quad \forall r>0
$$

is satisfied, then an ISS Lyapunov function for the overall system is given by

$$
V(x)=\max _{i=1, \ldots, n} \sigma_{i}^{-1}\left(V_{i}\left(x_{i}\right)\right) .
$$

If in addition each $\mu_{i}$ is additive in the external input, i.e., $\mu_{i}\left(s_{1}, \ldots, s_{n}, r\right)=\mu_{i}\left(s_{1}, \ldots, s_{n}, 0\right)+r$ and the small gain condition (9) is satisfied, then there exists a $\sigma \in \mathcal{K}_{\infty}^{n}$ as in Theorem 3.1 and an ISS Lyapunov function for the overall system is given by (13).

For the proofs of these theorems we refer to [4], [7], where they were given for some particular $\mu$. Proofs with a general $\mu$ are given in [9], [15], [16].

Note that by construction the Lyapunov function $V$ is not smooth, even if the functions $V_{i}$ for the subsystems are. However $V$ is Lipschitz continuous and hence differentiable almost everywhere. Methods of nonsmooth analysis [1], [2] can be used in this case.

\section{Applications}

In this section we give four examples illustrating the effectiveness of the small gain theorem stated in the previous section. The first example illustrates the construction method for an ISS-Lyapunov function in the relative simple case when the interconnection consists of linearly interconnected linear subsystems. Further we will consider the a CohenGrossberg neural network, an example related to formation control, and nonlinear transistor-linear resistor circuits.

\section{A. Linear systems}

Consider linear interconnected systems

$$
\Sigma_{i}: \dot{x}_{i}=A_{i} x_{i}+\sum_{j=1}^{n} \Delta_{i j} x_{j}+B_{i} u_{i}, \quad i=1, \ldots, n
$$

with $x_{i} \in \mathbb{R}^{N_{i}}, u_{i} \in \mathbb{R}^{M_{i}}$, and matrices $A_{i}, B_{i}$ of appropriate dimensions. Each system $\Sigma_{i}$ is ISS from $\left(x_{1}^{T}, \ldots, x_{i-1}^{T}, x_{i+1}^{T}, \ldots, x_{n}^{T}, u_{i}^{T}\right)^{T}$ to $x_{i}$ if and only if $A_{i}$ is Hurwitz. This can be seen for example with the Lyapunov function $V_{i}\left(x_{i}\right)=x_{i}^{T} P_{i} x_{i}$, where $P_{i}$ is a symmetric positive definite solution of $A_{i}^{T} P_{i}+P_{i} A_{i}=-Q_{i}$ for some symmetric positive definite matrix $Q_{i}$. It is known that there is a unique s.p.d. solution $P_{i}$ for any given s.p.d. $Q_{i}$ if and only if $A_{i}$ is Hurwitz. In that case, along trajectories of the autonomous system

$$
\dot{x}_{i}=A_{i} x_{i}
$$

we have

$$
\dot{V}_{i}=x_{i}^{T} P_{i} A_{i} x_{i}+x_{i}^{T} A_{i}^{T} P_{i} x_{i}=-x_{i}^{T} Q_{i} x_{i} \leq-c_{i}\left|x_{i}\right|^{2}
$$

for $c_{i}:=\lambda_{\min }\left(Q_{i}\right)>0$, the smallest eigenvalue of $Q$.
For the interconnected system (14) we obtain

$$
\begin{aligned}
\dot{V}_{i} & =2 x_{i}^{T} P_{i}\left(A_{i} x_{i}+\sum_{j \neq i} \Delta_{i j} x_{j}+B_{i} u_{i}\right) \\
& \leq-c_{i}\left|x_{i}\right|^{2}+2\left|x_{i}\right|\left\|P_{i}\right\|\left(\sum_{j \neq i}\left\|\Delta_{i j}\right\|\left|x_{j}\right|+\left\|B_{i}\right\|\left|u_{i}\right|\right) \\
& \leq-\varepsilon c_{i}\left|x_{i}\right|^{2}
\end{aligned}
$$

where the last inequality (15) is satisfied for some small $\varepsilon>0$ if

$$
\left|x_{i}\right| \geq \frac{2\left\|P_{i}\right\|}{c_{i}(1-\varepsilon)}\left(\sum_{j \neq i}\left\|\Delta_{i j}\right\|\left|x_{j}\right|+\left\|B_{i}\right\||u|\right)
$$

with $u:=\left(u_{1}^{T}, \ldots, u_{n}^{T}\right)^{T}$. To write this implication in the form of (6) we note that $\lambda_{\min }\left(P_{i}\right)\left|x_{i}\right|^{2} \leq V_{i}\left(x_{i}\right) \leq$ $\lambda_{\max }\left(P_{i}\right)\left|x_{i}\right|^{2}$. Let us denote $a_{i}^{2}=\lambda_{\min }\left(P_{i}\right), b_{i}^{2}=$ $\lambda_{\max }\left(P_{i}\right)$, the inequality (16) then is satisfied if

$V_{i}\left(x_{i}\right) \geq\left(\frac{2\left\|P_{i}\right\| b_{i}}{c_{i}(1-\varepsilon)}\right)^{2}\left(\sum_{j \neq i} \frac{\left\|\Delta_{i j}\right\|}{a_{j}} \sqrt{V_{j}\left(x_{j}\right)}+\left\|B_{i}\right\|\|u\|\right)^{2}$

Using the fact that for nonnegative real numbers $a, b$, we have $(a+b)^{2} \leq 2 a^{2}+2 b^{2}$, we see that the function $V_{i}$ is an ISS-Lyapunov function for $\Sigma_{i}$ with gains given by

$$
\gamma_{i j}(r)=\frac{2\left\|P_{i}\right\| b_{i}}{c_{i}(1-\varepsilon)} \frac{\left\|\Delta_{i j}\right\|}{a_{j}} \sqrt{r}
$$

for $i=1, \ldots, n, i \neq j$, and

$$
\gamma_{i u}(r)=2\left(\frac{2\left\|P_{i}\right\| b_{i}}{c_{i}(1-\varepsilon)}\left\|B_{i}\right\| r\right)^{2},
$$

for $i=1, \ldots, n$, and $r \geq 0$. Further we may take

$$
\mu_{i}(s, r)=2\left(\sum_{j=1}^{n} s_{j}\right)^{2}+r
$$

for $s \in \mathbb{R}_{+}^{n}$ and $r \in \mathbb{R}_{+}$. By defining $\gamma_{i i} \equiv 0$ for $i=1, \ldots, n$ we can write

$$
\bar{\Gamma}=\left(\begin{array}{ccccc}
0 & \gamma_{12} & \cdots & \gamma_{1 n} & \gamma_{1 u} \\
\gamma_{21} & \ddots & \cdots & \gamma_{2 n} & \gamma_{2 u} \\
\vdots & & \ddots & \vdots & \\
\gamma_{n 1} & \cdots & \gamma_{n n} & 0 & \gamma_{n u}
\end{array}\right)
$$

and have

$$
\begin{aligned}
& \bar{\Gamma}_{\mu}(s, r)= \\
& \left(\begin{array}{c}
2\left(\frac{2\left\|P_{1}\right\| b_{1}}{c_{1}(1-\varepsilon)}\right)^{2}\left(\sum_{j} \frac{\left\|\Delta_{1 j}\right\|}{a_{1}} \sqrt{s_{j}}\right)^{2}+2\left(\frac{2\left\|P_{1}\right\| b_{1}}{c_{1}(1-\varepsilon)}\left\|B_{1}\right\| r\right)^{2} \\
\vdots \\
\left(\frac{2\left\|P_{n}\right\| b_{n}}{c_{n}(1-\varepsilon)}\right)^{2}\left(\sum_{j} \frac{\left\|\Delta_{n j}\right\|}{a_{n}} \sqrt{s_{j}}\right)^{2}+2\left(\frac{2\left\|P_{n}\right\| b_{n}}{c_{n}(1-\varepsilon)}\left\|B_{n}\right\| r\right)^{2}
\end{array}\right)
\end{aligned}
$$

Interestingly, the choice of quadratic Lyapunov functions for the subsystems naturally leads to a nonlinear mapping $\bar{\Gamma}$. 
Proposition 4.1: Let each $\Sigma_{i}$ in (14) be ISS with a quadratic ISS-Lyapunov function $V_{i}$, so that the corresponding operator $\bar{\Gamma}_{\mu}$ can be taken as in (17). If the spectral radius of the associated matrix

$$
G=\left(\sqrt{2} \frac{2\left\|P_{i}\right\| b_{i}}{c_{i}(1-\varepsilon)} \frac{\left\|\Delta_{i j}\right\|}{a_{j}}\right)_{i j}
$$

is less than 1 , then the interconnection

$$
\Sigma: \quad \dot{x}=(A+\Delta) x+B u
$$

is ISS and its (nonsmooth) ISS-Lyapunov function can be taken as $V(x)=\max _{i} \frac{1}{\hat{s}_{i}} x_{i}^{\top} P_{i} x_{i}$ for some positive vector $\hat{s} \in \mathbb{R}_{+}^{n}$.

Proof: If the spectral radius of $G$ is less than one, then there exists a positive vector $\tilde{s}$ satisfying $G \tilde{s}<\tilde{s}$ : Just add a small $\delta>0$ to every entry of $G$, so that the spectral radius $\rho(\tilde{G})$ of $\tilde{G}=G+\delta$ is still less than one, due to continuity of the spectrum. Then there exists a Perron-Frobenius vector $\tilde{s}$ such that $G \tilde{s}<\tilde{G} \tilde{s}=\rho(\tilde{G}) \tilde{s}<\tilde{s}$.

Now define $\hat{s}$ by $\hat{s}_{i}=\tilde{s}_{i}^{2}$ for $i=1, \ldots, n$. We claim that the straight half-line spanned by this vector $\hat{s}$ in the positive orthant is an $\Omega$-path for $\Gamma_{\mu}$.

Indeed, for all $r>0$ we have

$$
\begin{aligned}
\Gamma_{\mu}(\hat{s} r)_{i} & =2\left(\sum_{j} \frac{2\left\|P_{i}\right\| b_{i}}{c_{i}(1-\varepsilon)} \frac{\left\|\Delta_{i j}\right\|}{a_{j}} \sqrt{\hat{s}_{j} r}\right)^{2} \\
& =\left(\sum_{j} \sqrt{2} \frac{2\left\|P_{i}\right\| b_{i}}{c_{i}(1-\varepsilon)} \frac{\left\|\Delta_{i j}\right\|_{a_{j}}}{a_{j}}\right)^{2} \cdot r \\
& <\left(\tilde{s}_{i}\right)^{2} r=\hat{s}_{i} r .
\end{aligned}
$$

The existence of an operator $\tilde{D}$ is now straightforward. By Theorem 3.2 an ISS-Lyapunov function can be taken as $V(x)=\max _{i} \frac{1}{\hat{s}_{i}} x_{i}^{\top} P_{i} x_{i}$.

A different approach to the stability analysis of interconnected linear systems has been undertaken in [13], [10], where stability conditions for interconnected systems have been derived and where the problem of constructing quadratic Lyapunov functions for the interconnected system is investigated.

\section{B. Neural networks}

Consider a Cohen-Grossberg neural network, see [22], e.g., given by

$$
\dot{x}_{i}(t)=-a_{i}\left(x_{i}(t)\right)\left(b_{i}\left(x_{i}(t)\right)-\sum_{j=1}^{n} t_{i j} s_{j}\left(x_{j}(t)\right)+J_{i}\right),
$$

$i=1, \ldots, n, n \geq 2$, where $x_{i}$ denotes the state of the $i$ th neuron, $a_{i}$ is a strictly positive amplification function, $b_{i}$ typically has the same sign as $x_{i}$ and is assumed to satisfy $\left|b_{i}\left(x_{i}\right)\right|>\tilde{b}_{i}\left(\left|x_{i}\right|\right)$ for some $\tilde{b}_{i} \in \mathcal{K}_{\infty}$, the activation function $s_{i}$ is typically assumed to be sigmoid. The matrix $T=\left(t_{i j}\right)_{i, j=1, \ldots, n}$ describes the interconnection of neurons in the network and $J_{i}$ is a given constant input from outside. However for our consideration we allow $J_{i}$ to be an arbitrary measurable function in $L_{\infty}$.
Note that for any sigmoid function there exists a $\gamma_{i} \in \mathcal{K}$ such that $\left|s_{i}\left(x_{i}\right)\right|<\gamma_{i}\left(\left|x_{i}\right|\right)$, following [22] we assume $0<$ $\underline{\alpha}_{i}<a_{i}\left(x_{i}\right)<\bar{\alpha}_{i}$.

Recall the triangle inequality for $\mathcal{K}_{\infty}$-functions: For any $\gamma, \rho \in \mathcal{K}_{\infty}$ and any $a, b \geq 0$ it holds

$$
\gamma(a+b) \leq \gamma \circ(\mathrm{id}+\rho)(a)+\gamma \circ\left(\mathrm{id}+\rho^{-1}\right)(b) .
$$

Define $V_{i}\left(x_{i}\right)=\left|x_{i}\right|$ then each subsystem is ISS since the following implication holds by the triangle inequality

$$
\begin{gathered}
\left|x_{i}\right|>\tilde{b}_{i}^{-1} \circ(\mathrm{id}+\rho)\left(\frac{\bar{\alpha}_{i}}{\underline{\alpha}_{i}-\varepsilon} \sum_{j=1}^{n}\left|t_{i j}\right| \gamma_{j}\left(\left|x_{j}\right|\right)\right) \\
+\tilde{b}_{i}^{-1} \circ\left(\mathrm{id}+\rho^{-1}\right)\left(\frac{\bar{\alpha}_{i}}{\underline{\alpha}_{i}-\varepsilon}\left|J_{i}\right|\right)
\end{gathered}
$$

$$
>\tilde{b}_{i}^{-1}\left(\frac{\bar{\alpha}_{i}}{\underline{\alpha}_{i}-\varepsilon}\left(\sum_{j=1}^{n}\left|t_{i j}\right| \gamma_{j}\left(\left|x_{j}\right|\right)+\left|J_{i}\right|\right)\right) \Longrightarrow
$$

$$
\begin{aligned}
\dot{V}_{i}=-a_{i}\left(x_{i}\right)\left(\left|b_{i}\left(x_{i}\right)\right|\right. & \left.-\operatorname{sign} x_{i} \sum_{j=1}^{n} t_{i j} s_{j}\left(x_{j}\right)+\operatorname{sign} x_{i} J_{i}\right) \\
& <-\varepsilon\left|b_{i}(x)\right|
\end{aligned}
$$

for some $\varepsilon$ satisfying $\underline{\alpha}_{i}>\varepsilon>0$ and arbitrary function $\rho \in \mathcal{K}_{\infty}$.

In this case we have

$\mu_{i}(s, r)=\tilde{b}_{i}^{-1} \circ(\mathrm{id}+\rho)\left(s_{1}+\cdots+s_{n}\right)+\tilde{b}_{i}^{-1} \circ\left(\mathrm{id}+\rho^{-1}\right)(r)$

additive with respect to the external inputs and

$$
\gamma_{i j}=\frac{\bar{\alpha}_{i}\left|t_{i j}\right|}{\underline{\alpha}_{i}-\varepsilon} \gamma_{j}\left(\left|x_{j}\right|\right), \quad \gamma_{i u}=\frac{\bar{\alpha}_{i} \text { id }}{\underline{\alpha}_{i}-\varepsilon} .
$$

Note that so far we have not imposed any restrictions on the coefficients $t_{i j}$. Moreover the assumptions imposed on $a_{i}, b_{i}, s_{i}$ are essentially milder then in [22]. However to obtain the ISS property of the network we need to require more, i.e., a small gain condition has to be met, which imposes restrictions on the coupling terms $t_{i j} s_{j}\left(x_{j}\right)$.

Theorem 4.2: Let $\Gamma_{\mu}$ be given by $\gamma_{i j}$ and $\mu_{i}, i, j=$ $1, \ldots, n$ as calculated above for the Cohen-Grossberg neural network (19). Assume $\Gamma_{\mu}$ satisfies the small gain condition (9). Then the Cohen-Grossberg neural network is ISS from $\left(J_{1}, \ldots, J_{n}\right)^{T}$ to $x$.

Remark 4.3: In [22] the authors have proved that there exists a unique equilibrium point for the network and given constant external inputs. They also have proved the exponential stability of this equilibrium. We have considered arbitrary external inputs in the network and proved the ISS property for the interconnection. 


\section{Formation control}

In [21] formations of vehicles on the plane as in Figure 1 have been considered. Using feedback linearization local controllers have been designed that render the formation error between two consecutive vehicles input-to-state stable with respect to the up-link formation error. Since cascades of ISS systems are ISS it could be shown that cascades of vehicles are "leader-to-formation" stable (ISS).

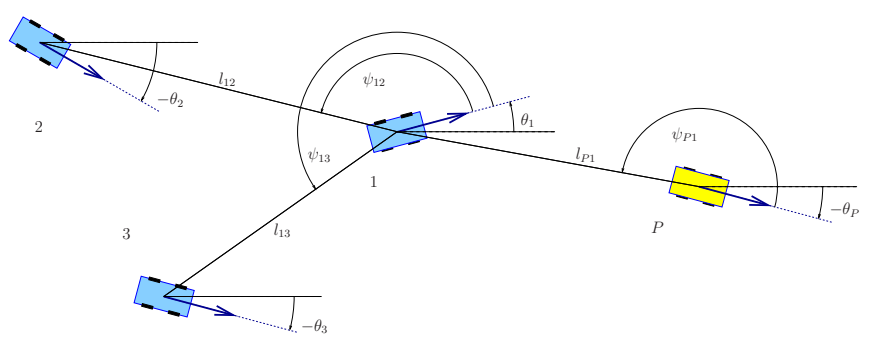

Fig. 1. A formation of four vehicles (1-3) following a designated leader (P).

Due to the converse ISS Lyapunov theorems, it is clear that there has to exist an ISS Lyapunov function for the entire formation error; here we show how to find it.

The dynamics for each vehicle $i$ in Figure 1 is given by

$$
\dot{x}_{i}=v_{i} \cos \theta_{i}, \quad \dot{y}_{i}=v_{i} \sin \theta_{i}, \quad \dot{\theta}_{i}=w_{i},
$$

$\left(x_{i}, y_{i}, \theta_{i}\right)$ being the absolute position and orientation of the $i$ th vehicle. In the following the modelling parameter $d$ denotes the distance from the wheel axis to the reference point on each vehicle. The control inputs $\left(v_{i}, w_{i}\right)$ are the translational and rotational velocity. The separation distance between two consecutive vehicles, a leader $i$ and a follower $j$, is denoted by $l_{i j}$, and the relative bearing between them is $\psi_{i j}$. For both values constant specification parameters $l_{i j}^{s p e c}, \psi_{i j}^{\text {spec }}$ are fixed and describe the formation. The formation error is

$$
\tilde{z}_{i j}:=\left(\tilde{l}_{i j}, \tilde{\psi}_{i j}\right)^{\top}:=\left(l_{i j}^{s p e c}-l_{i j}, \psi_{i j}^{s p e c}-\psi_{i j}\right)^{\top} .
$$

The control objective is to drive $\tilde{z}_{i j}$ to 0 . In [21] a controller is proposed that renders $\tilde{z}_{i j}$ ISS with respect to $\tilde{z}_{k i}$, where $k$ denotes the number of the vehicle in front of vehicle $i$. It is shown that the Lyapunov function candidate

$$
V_{i j}\left(\tilde{z}_{i j}\right)=\frac{1}{2 k_{1}^{j}}\left|\tilde{l}_{i j}\right|^{2}+\frac{1}{2 k_{2}^{j}}\left|\tilde{\psi}_{i j}\right|^{2}
$$

satisfies the implication

$$
\begin{array}{r}
\left|\tilde{z}_{i j}\right| \geq \frac{\max \left\{k_{1}^{i}, k_{2}^{i}\right\}\left(d+l_{k i}^{s p e c}+\left|\tilde{z}_{k i}\right|\right)\left|\tilde{z}_{k i}\right|}{(1-\varepsilon) d \min \left\{k_{1}^{j}, k_{2}^{j}\right\}} \\
\Longrightarrow \dot{V}_{i j} \leq-\varepsilon\left|\tilde{z}_{i j}\right|^{2}
\end{array}
$$

where $K^{i}=\left(k_{1}^{i}, k_{2}^{i}\right)^{T}$ is the feedback gain used in the feedback linearization construction of the controller. Impli- cation (23) is implied by

$$
\begin{aligned}
& V_{i i}\left(\tilde{z}_{i j}\right) \geq \\
& \left(\frac{\max \left\{k_{1}^{i}, k_{2}^{i}\right\}\left(d+l_{k i}^{s p e c}+\sqrt{V_{k i}\left(\tilde{z}_{k i}\right)}\right) \sqrt{V_{k i}\left(\tilde{z}_{k i}\right)}}{\sqrt{2} \min \left\{\sqrt{k_{1}^{i}}, \sqrt{k_{2}^{i}}\right\}(1-\varepsilon) d \min \left\{k_{1}^{j}, k_{2}^{j}\right\}}\right)^{2} \\
& \Longrightarrow \dot{V}_{i j} \leq-\varepsilon\left|\tilde{z}_{i j}\right|^{2} .
\end{aligned}
$$

which is in the form of (6). For a cascade formation of $n$ vehicles the gain matrix takes the form

$$
\Gamma=\left(\begin{array}{cccc}
0 & \ldots & \ldots & 0 \\
\gamma_{21} & 0 & & \vdots \\
\vdots & \ddots & \ddots & \vdots \\
\gamma_{n 1} & \ldots & \gamma_{n, n-1} & 0
\end{array}\right)
$$

where $\gamma_{i j}$ is given according to (24) and $\mu_{i}$ is summation for $i=1, \ldots, n$. Observe that if each vehicle follows exactly one other vehicle, each row except the first one in $\Gamma$ contains at exactly one nonzero element. An $\Omega$-path $\sigma=\left(\sigma_{1}, \ldots, \sigma_{n}\right)^{\top} \in \mathcal{K}_{\infty}^{n}$ can now iteratively constructed as follows: Pick $\sigma_{1}=\mathrm{id} \in \mathcal{K}_{\infty}$. Iteratively, for $i=2, \ldots, n$, choose $\sigma_{i} \in \mathcal{K}_{\infty}$ such that $\sum_{j=1}^{i-1} \gamma_{i j} \circ \sigma_{j}<\sigma_{i}$. Clearly that is always possible, as a sum of $\mathcal{K}_{\infty}$ functions can always be bounded from above by a $\mathcal{K}_{\infty}$ function.

The ISS Lyapunov function for the formation error is now given according to formula (13).

\section{Transistor networks}

In [14] a nonlinear transistor-linear resistor model is considered, which is characterized by the set of equations

$$
\dot{z}_{i}+A_{i} f_{i}\left(z_{i}\right)+B_{i} g_{i}\left(z_{i}\right)=b_{i}(t), \quad i=1, \ldots, n,
$$

where $z_{i} \in \mathbb{R}^{N_{i}}, A_{i}=\left(a_{k l}^{i}\right), B_{i}=\left(b_{k j}^{i}\right)$ are constant square matrices with $a_{k k}^{i}>0$ and $b_{k k}^{i}>0$, where $f_{i}, g_{i}$ : $\mathbb{R}^{N_{i}} \rightarrow \mathbb{R}^{N_{i}}$ are continuously differentiable in $z_{i}$, satisfy $f_{i}\left(z_{i}\right)=0$ and $g_{i}\left(z_{i}\right)=0$ if and only if $z_{i}=0$, and further it may be assumed that in fact $f_{k}^{i}\left(z_{i}\right)=f_{k}^{i}\left(z_{k}^{i}\right)$ and $g_{k}^{i}\left(z_{i}\right)=g_{k}^{i}\left(z_{k}^{i}\right)$ where $f_{i}\left(z_{i}\right)=\left(f_{1}^{i}\left(z_{i}\right), \ldots, f_{N_{i}}^{i}\left(z_{i}\right)\right)^{\top}$, $g_{i}\left(z_{i}\right)=\left(g_{1}^{i}\left(z_{i}\right), \ldots, g_{N_{i}}^{i}\left(z_{i}\right)\right)^{\top}$, and $z_{i}=\left(z_{1}^{i}, \ldots, z_{N_{i}}^{i}\right)^{\top}$. As in [17] it is assumed that $f_{k}^{i}\left(z_{k}^{i}\right) / z_{k}^{i} \geq \delta>0$ and $g_{k}^{i}\left(z_{k}^{i}\right) / z_{k}^{i} \geq \delta$ for all $z_{k}^{i} \neq 0$ and that $\partial / \partial z_{k}^{i} f_{k}^{i}(0) \geq \delta$ and $\partial / \partial z_{k}^{i} f_{k}^{i}(0) \geq \delta$. As in [14] we content ourselves with the case

$$
b_{i}(t) \equiv 0, \quad i=1, \ldots, n .
$$

The interconnection between the subsystems (26) is described via terms $C_{i j} g_{j}\left(z_{j}\right)$, where $C_{i j}$ are constant matrices of appropriate dimensions, leading to subsystem dynamics

$$
\dot{z}_{i}+A_{i} f_{i}\left(z_{i}\right)+B_{i} g_{i}\left(z_{i}\right)+\sum_{j \neq i} C_{i j} g_{j}\left(z_{j}\right)=0, \quad i=1, \ldots, n \text {. }
$$

This may be written as

$$
\dot{z}+A f(z)+B g(z)=0
$$

where $z^{\top}=\left(z_{1}^{\top}, \ldots, z_{n}^{\top}\right), f^{\top}=\left(f_{1}^{\top}, \ldots, f_{n}^{\top}\right), g^{\top}=$ $\left(g_{1}^{\top}, \ldots, g_{n}^{\top}\right), A$ accommodates the matrices $A_{i}$ on the 
diagonal, and $B$ accommodates the matrices $B_{i}$ and $C_{i j}$ in the obvious manner.

Due to the nonlinear terms $f_{i}$ a quadratic Lyapunov function $V_{i}\left(z_{i}\right)=z^{\top} P_{i} z_{i}$ with $P A_{i}+A_{i}^{\top} P_{i}=-Q_{i}$ for some positive definite matrix $Q_{i}$ bears some difficulties when it comes to consider the interconnection structure and to derive gains between the subsystems. Instead, we decompose system (28) into its scalar subsystems

$$
\begin{aligned}
\dot{\tilde{z}}_{\lambda}= & -a_{\lambda \lambda} \tilde{f}_{\lambda}\left(\tilde{z}_{\lambda}\right)-b_{\lambda \lambda} \tilde{g}_{\lambda}\left(\tilde{z}_{\lambda}\right) \\
& -\sum_{\nu \neq \lambda}\left(a_{\lambda \nu} \tilde{f}_{\nu}\left(\tilde{z}_{\nu}\right)+b_{\lambda \nu} \tilde{g}_{\nu}\left(\tilde{z}_{\nu}\right)\right),
\end{aligned}
$$

for $\lambda=1, \ldots, N=\sum_{i} N_{i}, A=\left(a_{\lambda \nu}\right), B=\left(b_{\lambda \nu}\right)$ and where $\tilde{f}_{\lambda}$ and $\tilde{g}_{\lambda}$ denote the component functions of $f$ and $g$, respectively, and $z$ is decomposed into $\tilde{z}_{1}, \ldots, \tilde{z}_{N}$.

Using a small $\varepsilon>0$, standard estimates, and the Lyapunov function candidates $V_{\lambda}\left(\tilde{z}_{\lambda}\right)=\frac{1}{2} \tilde{z}_{\lambda}^{2}$, we arrive at

$$
\dot{V}_{\lambda} \leq-\left(a_{\lambda \lambda}+b_{\lambda \lambda}\right) \delta \varepsilon \tilde{z}_{\lambda}^{2}
$$

if

$$
\left|\tilde{z}_{\lambda}\right| \geq \sum_{\nu \neq \lambda} \tilde{\gamma}_{\lambda \nu}\left(\left|\tilde{z}_{\nu}\right|\right)
$$

where

$$
\begin{aligned}
\tilde{\gamma}_{\lambda \nu}(s)=\frac{1}{(1-\varepsilon) \delta\left(a_{\lambda \lambda}+b_{\lambda \lambda}\right)} & \quad\left[a_{\lambda \nu} \max \left\{\tilde{f}_{\nu}(s),-\tilde{f}_{\nu}(-s)\right\}\right. \\
& \left.+b_{\lambda \nu} \max \left\{\tilde{g}_{\nu}(s),-\tilde{g}_{\nu}(-s)\right\}\right] .
\end{aligned}
$$

Now we note that due to the properties of the functions $\tilde{f}_{\lambda}$ and $\tilde{g}_{\lambda}$, the function $\tilde{\gamma}_{\lambda \nu}$ is of class $\mathcal{K}_{\infty}$ or equals constantly zero. Hence for the functions $\gamma_{\lambda \nu}$ given by $\gamma_{\lambda \nu}(s)=$ $\tilde{\gamma}_{\lambda \nu}(\sqrt{2 s})$, we have

$$
\begin{gathered}
V_{\lambda}\left(\tilde{z}_{\lambda}\right) \geq \frac{1}{2}\left(\sum_{\nu \neq \lambda} \gamma_{\lambda \nu}\left(V_{\nu}\left(\tilde{z}_{\nu}\right)\right)\right)^{2} \\
\Longrightarrow \dot{V}_{\lambda} \leq-2\left(a_{\lambda \lambda}+b_{\lambda \lambda}\right) \delta \varepsilon \tilde{z}_{\lambda}^{2}
\end{gathered}
$$

i.e., the defining implication form (6) for an ISS Lyapunov function.

Now, if the small gain condition is satisfied for $\Gamma=\left(\gamma_{\lambda \nu}\right)$ and $\mu_{\lambda}(s)=\frac{1}{2}\left(\sum_{\nu} s_{\nu}\right)^{2}$, then the trivial solution of (28) is globally asymptotically stable, and a Lyapunov function is given by (13).

\section{CONCLUSIONS}

In this paper we have considered several applications of the general ISS Lyapunov small gain theorem. In particular we have shown how ISS Lyapunov functions can be constructed in a bottom-to-top design fashion for linear systems, neural networks, vehicle formation control systems, and for transistor networks.

\section{ACKNOWLEDGMENTS}

This research has been supported by the German Research Foundation (DFG) as part of the Collaborative Research
Center 637 "Autonomous Cooperating Logistic Processes". B. S. Rüffer has been supported by the Australian Research Council under grant DP0771131.

\section{REFERENCES}

[1] F. H. Clarke. Nonsmooth analysis in control theory: a survey. European J. Control, 7:63-78, 2001.

[2] F. H. Clarke, Yu. S. Ledyaev, R. J. Stern, and P. R. Wolenski. Nonsmooth analysis and control theory. Springer-Verlag, New York, 1998.

[3] S. Dashkovskiy, B. Rüffer, and F. Wirth. A small-gain type stability criterion for large scale networks of ISS systems. In Proc. of 44th IEEE Conference on Decision and Control and European Control Conference CDC/ECC 2005, pages 5633 - 5638, Seville, Spain, December 2005.

[4] S. Dashkovskiy, B. Rüffer, and F. Wirth. An ISS Lyapunov function for networks of ISS systems. In Proc. 17th Int. Symp. Math. Theory of Networks and Systems, MTNS2006, pages 77-82, Kyoto, Japan, July 24-28 2006

[5] S. Dashkovskiy, B. Rüffer, and F. Wirth. An ISS small-gain theorem for general networks. Math. Control Signals Systems, 19:93-122, 2007.

[6] S. Dashkovskiy, B. Rüffer, and F. Wirth. Numerical verification of local input-to-state stability for large netwoks. In Proc. of 46th IEEE Conference on Decision and Control, CDC 2007, pages 4471 - 4476, 12-14 December, 2007, New Orleans, USA, December 2007.

[7] S. Dashkovskiy, B. S. Rüffer, and F. R. Wirth. A Lyapunov ISS small gain theorem for strongly connected networks. In Proc. 7th IFAC Symposium on Nonlinear Control Systems, NOLCOS2007, pages 283288, Pretoria, South Africa, August 2007.

[8] S. Dashkovskiy, B. S. Rüffer, and F. R. Wirth. Stability of autonomous logistic processes via small gain criteria. In Proc. International Conference on Dynamics in Logistics, pages 359-366, Bremen, August 2007.

[9] S. N. Dashkovskiy, B. S. Rüffer, and F. R. Wirth. Construction of an iss-lyapunov function for large-scale interconnections of iss systems. In preparation, 2008.

[10] D. Hinrichsen and A. J. Pritchard. Composite systems with uncertain couplings of fixed structure: Scaled riccati equations and the problem of quadratic stability. SIAM J. Control Optim., 2008. to appear.

[11] Z.-P. Jiang, I. M. Y. Mareels, and Y. Wang. A Lyapunov formulation of the nonlinear small-gain theorem for interconnected ISS systems. Automatica J. IFAC, 32(8):1211-1215, 1996.

[12] Z.-P. Jiang, A. R. Teel, and L. Praly. Small-gain theorem for ISS systems and applications. Math. Control Signals Systems, 7(2):95120, 1994.

[13] M. Karow, D. Hinrichsen, and A. J. Pritchard. Spectral value sets, stability radii and $\mu$-functions for composite systems. SIAM J. Control Optim., 45(3):846-884, 2006.

[14] A. N. Michel, R. K. Miller, and W. Tang. Lyapunov stability of interconnected systems: decomposition into strongly connected subsystems. IEEE Trans. Circuits and Systems, 25(9):799-809, 1978

[15] B. S. Rüffer. Monotone Systems, Graphs, and Stability of Large-Scale Interconnected Systems. Dissertation, Fachbereich 3, Mathematik und Informatik, Universität Bremen, Germany, August 2007. Available online: http://nbn-resolving.de/urn:nbn:de:gbv:46-diss000109058.

[16] B. S. Rüffer. Monotone inequalities, dynamical systems, and paths in the positive orthant of euclidean $n$-space. Positivity, 2008. submitted.

[17] I. W. Sandberg. Some theorems on the dynamic response of nonlinear transistor networks. Bell System Tech. J., 48:35-54, 1969.

[18] R. Sepulchre, M. Janković, and P. V. Kokotović. Constructive nonlinear control. Communications and Control Engineering Series. Springer-Verlag, Berlin, 1997.

[19] Eduardo D. Sontag. Smooth stabilization implies coprime factorization. IEEE Trans. Automat. Control, 34(4):435-443, 1989.

[20] Eduardo D. Sontag and Yuan Wang. New characterizations of inputto-state stability. IEEE Trans. Automat. Control, 41(9):1283-1294, 1996.

[21] H. G. Tanner, G. J. Pappas, and V. Kumar. Leader-to-formation stability. IEEE Transactions on Robotics and Automation, 20(3):443455, 2004.

[22] Lin Wang and Xingfu Zou. Exponential stability of Cohen-Grossberg neural networks. Neural networks, 15:415-422, 2002. 